\title{
Assessing the Short-Term Efficacy and Safety of Guselkumab for Moderate-to-Severe Plaque Psoriasis: Meta-Analysis of Randomized Controlled Trials
}

\author{
Jing Yang, Zongming Wang $₫$, and Xilin Zhang \\ Department of Dermatology and Venereology, Chong Qing Three Gorges Central Hospital, Chongqing 404100, China \\ Correspondence should be addressed to Zongming Wang; zm09000@126.com
}

Received 16 April 2020; Accepted 11 June 2020; Published 17 July 2020

Academic Editor: Luis Alberto Ponce-Soto

Copyright ( 2020 Jing Yang et al. This is an open access article distributed under the Creative Commons Attribution License, which permits unrestricted use, distribution, and reproduction in any medium, provided the original work is properly cited.

\begin{abstract}
Background. To investigate the efficacy and safety of guselkumab in the treatment of moderate-to-severe plaque psoriasis. Methods. A systematic review was undertaken to identify double-blind randomized controlled trials (RCTs). PubMed, Web of Science, Cochrane Library, EMBASE, and Google Scholar databases were searched before 1 March 2020. The odds ratios (ORs) with 95\% confidence interval (CI) were calculated. All analyses were conducted with intention-to-treat basis. A range of sensitivity analyses was undertaken. Results. A total of 7 articles contained 1206 plaque psoriasis patients with guselkumab, 585 patients with placebo, and 1250 patients with adalimumab were included. The results indicated that guselkumab had better efficacy than placebo or adalimumab for Psoriasis Area and Severity Index score reductions from baseline of 75\% (PASI 75) (OR =61.37, $95 \% \mathrm{CI}=31.15$ to $120.91 ; \mathrm{OR}=3.08,95 \% \mathrm{CI}=2.35$ to 4.06 ), Investigator's Global Assessment scores of 0 or 1 (IGA $0 / 1$ ) $(\mathrm{OR}=65.75,95 \% \mathrm{CI}=45.54$ to $94.95 ; \mathrm{OR}=2.79,95 \% \mathrm{CI}=2.17$ to 3.59$)$, and Dermatology Life Quality Index scores of 0 or 1 (DLQI $0 / 1)(\mathrm{OR}=29.64,95 \% \mathrm{CI}=18.80$ to 46.73 ; $\mathrm{OR}=1.86,95 \% \mathrm{CI}=1.50$ to 2.31$)$. The guselkumab had similar safety with placebo or adalimumab about the incidence of adverse events (AEs) $(\mathrm{OR}=1.05,95 \% \mathrm{CI}=0.86$ to $1.29 ; \mathrm{OR}=0.97,95 \% \mathrm{CI}=$ 0.79 to 1.19$)$ and serious adverse events (SAEs) $(\mathrm{OR}=1.03,95 \% \mathrm{CI}=0.47$ to $2.27 ; \mathrm{OR}=0.91,95 \% \mathrm{CI}=0.44$ to 1.87 ). Meanwhile, there was no statistically significant association of infections and serious infections compared with the placebo or adalimumab group. The guselkumab was more effective and had the similar tolerance. Conclusion. The guselkumab had excellent efficacy and great safety in moderate-to-severe plaque psoriasis, but long-term safety remained to be determined.
\end{abstract}

\section{Introduction}

Psoriasis is a chronic immune-mediated inflammatory skin disease that manifests in the skin or joint [1]. It affects nearly $1 \sim 3 \%$ of the population worldwide and has brought enormous financial pressure to the patients [2]. The etiology of psoriasis is unknown; it may be associated with the defect in proliferation and differentiation of keratinocytes and inflammatory cell infiltration. The subtypes of psoriasis are diverse; $90 \%$ of the total number of patients are plaque psoriasis $[3,4]$. The lesions of plaque psoriasis show clear red plaques, which are covered with silvery white scales and accompanied by obviously itching symptoms. The main characteristics of plaque psoriasis are infiltrative plaques and thicker scales with light red to dark red. If the scales are scraped out, a thin film phenomenon and spotted bleeding could be seen. They usually have a longer course [5].

At present, the main inhibitors for the treatment of psoriasis are IL-23 inhibitors, IL-17 inhibitors, and TNF- $\alpha$ inhibitors. The central role of interleukin-23/interleukin-17 (IL-23/IL-17) axis in the pathogenesis of psoriasis and the effectiveness of its targeted therapy have been confirmed by numerous studies $[6,7]$. IL-23 belongs to the IL-12 cytokine family. It is a heterodimer composed of $\mathrm{p} 40$ and $\mathrm{p} 19$ subunits [8]. Guselkumab is a fully human immunoglobulin $\mathrm{G} 1 \lambda$ (IgG $1 \lambda$ ) monoclonal antibody that blocks the downstream signaling of IL-23 by specifically binding to the p19 subunit of IL-23 [9]. As a proinflammatory factor, TNF- $\alpha$ is produced by a variety of skin immune cells and could regulate the production of IL-23. At the same time, they cooperate 
with IL-17 to promote keratinocytes to express various psoriasis-related inflammatory factors. Therefore, TNF- $\alpha$ inhibitors have shown remarkable effects in the treatment of plaque psoriasis. Adalimumab is the first successfully developed fully human IgG, which has a high affinity for soluble TNF- $\alpha$, and could effectively counteract the biological function of TNF- $\alpha$ by blocking the interaction between TNF- $\alpha$ and its receptors P55 and P75. Thus, the condition of psoriasis patients has been improved [10]. Currently, the guselkumab was in the phase III clinical trials for the treatment of moderate-to-severe plaque psoriasis and the phase II clinical trials for the treatment of arthritis psoriasis. The adalimumab was in the phase III clinical trial for the treatment of psoriasis. Relevant clinical trials of guselkumab showed that the Psoriasis Area and Severity Index (PASI) scores were decreased significantly after treatment and showed good safety [11-13]. Kim et al. [14] indicated that adalimumab treatment for moderate to severe plaque psoriasis was associated with greater PASI reduction, higher rates of resolution of skin signs and symptoms, and greater improvements in dermatological life quality. The studies showed that the effects of anti-IL-23p19 inhibitors were better than those of the IL-17A inhibitors, and they had a shorter induction period and a lower loading dose [15].

Many studies have proved that guselkumab was effective and safe, but some results showed inconsistent conclusions. Gordon et al. [16]. indicated that the infection rate of guselkumab was higher than that of placebo or adalimumab, which was different from other studies. Additionally, there was no study or analysis comparing the efficacy or safety of guselkumab with placebo or adalimumab. This meta-analysis is the first comprehensive analysis of the efficacy and safety of guselkumab, so as to provide further reliable basis for clinical application.

\section{Materials and Methods}

2.1. Study Identification. The electronic databases including PubMed, Web of Science, Cochrane Library, EMBASE, and Google Scholar databases were searched from 1 January 2000 to 1 January 2020 for studies published in English. The double-blind randomized controlled trials (RCTs) investigating the efficacy and safety of guselkumab were systematically retrieved. Keywords and search strategy were as follows: "IL-23 inhibitor" or "IL-23" or "IL-23p19" or "antiIL-23" or "guselkumab" or "CNTO1959" combined with "psoriasis." Comments, editorials, and letters were removed. In addition, the references of these articles were also screened to find other relevant articles. The search strategy is shown in Figure 1.

2.2. Study Selection. Trials were selected based on the following inclusion criteria: (1) the study design was limited to double-blind, randomized, placebo-controlled trials; (2) the patients were all older than 18 years, and they had stable ( $\geq 6$ months) moderate-to-severe chronic plaque at baseline with Body Surface Area (BSA) involvement of $10 \%$ or greater; (3) the studies should provide at least one efficacy outcome for short-term treatment: the reduction from base- line in the Psoriasis Area and Severity Index 75 (PASI 75), Investigator's Global Assessment scores of 0 or 1 (IGA 0/1), or Dermatology Life Quality Index scores of 0 or 1 (DLQI $0 / 1)$; (4) the studies should provide at least one safety outcome for short-term treatment: one or more adverse events (AEs) and one or more serious adverse events (SAEs); (5) the follow-up time was 16 or 24 weeks. The exclusion criteria were as follows: (1) the patients with psoriasis who were under 18 years of age; (2) the patients of active inflammatory diseases that could have interfered with study assessments who were ineligible, for example, drug-induced psoriasis and guttate, erythrodermic, or pustular psoriasis; (3) the women who were pregnant, breastfeeding, or planning to become pregnant; (4) the patients who had had prior exposure to the study drug or undergone major surgery 12 weeks or less before randomization, and the surgery was planned within 12 months after screening; (5) the patients who had history of allergy or hypersensitivity to a systematically administrated biologic agent; (6) the case-control studies, cohort studies, review articles, conference abstracts, case reports, and unpublished articles.

2.3. Data Abstraction and Quality Assessment. Two researchers independently extracted the following information from each study: study design, baseline patient characteristics, interventions, national clinical trial number, IGA, BSA, PASI, and DLQI. The efficacy parameters were PASI 75, PASI 90, PASI 100, IGA 0/1, and DLQI 0/1. The safety parameters were AEs, SAEs, and infections and serious infections. And the PASI 75, IGA 0/1, and DLQI 0/1 were primary indices; the other parameters were secondary indices. The AEs, SAEs, and infections and serious infections were safety indices. The methodological quality of included studies was assessed by one independent reviewer. Any disagreements were discussed with the third researcher.

2.4. Statistical Analysis. The efficacy and safety of guselkumab were assessed and compared with a placebo or adalimumab. We performed meta-analysis to calculate odds ratios (ORs) and 95\% CIs using the Mantel-Haenszel statistical method. A random effects model was used to pool the data, and $I^{2}$ statistic was evaluated heterogeneity between summary data. Sensitivity analysis was performed by excluding low-quality studies. All meta-analyses were performed using RevMan version 5.3. All tests were 2 -tailed, and $P<0.05$ was considered statistically significant.

\section{Results}

3.1. Literature Search and Study Characteristics. From the searches for studies, 5724 potentially eligible records were identified. Titles and abstracts of these articles were screened for inclusion. Finally, 7 articles that contained 1206 plaque psoriasis patients with guselkumab, 585 patients with placebo, and 1250 patients with adalimumab were included. The process of study selection is shown in Figure 1. The characteristics of enrolled studies are represented detailedly in Table 1. 


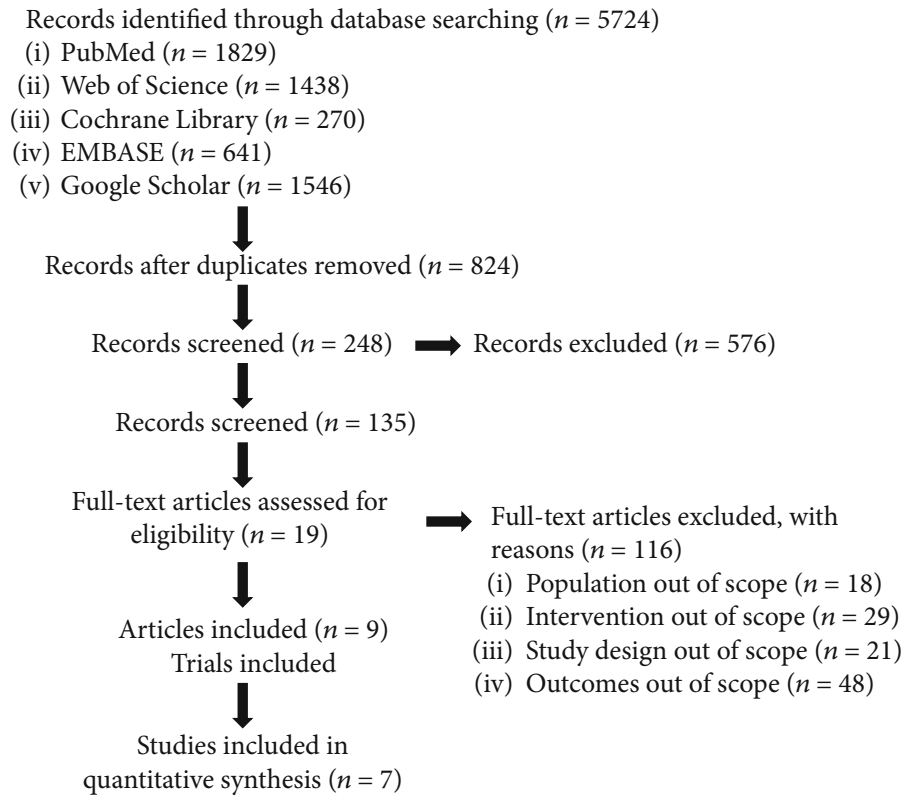

Figure 1: Flowchart of study selection.

3.2. Risk of Bias. The methodological quality for the included studies was assessed independently by two researchers based on Cochrane risk-of-bias criteria, and each quality item was graded as low risk, high risk, or unclear risk. The 7 items used to evaluate bias in each trial included (1) random sequence generation, (2) allocation concealment, (3) blinding of participants and personnel, (4) blinding of outcome assessment, (5) incomplete outcome data, (6) selective reporting, and (7) other bias. Overall, the risk of bias for most of the studies was judged to be low (Figure 2).

3.3. Primary Outcomes. All tests were conducted using a random effects model. As shown in Figure 3, there were significant differences in PASI $75(\mathrm{OR}=61.37,95 \% \mathrm{CI}=31.15$ to 120.91), IGA $0 / 1(\mathrm{OR}=65.75,95 \% \mathrm{CI}=45.54$ to 94.95$)$, and DLQI $0 / 1 \quad(\mathrm{OR}=29.64,95 \% \mathrm{CI}=18.80$ to 46.73$)$ between the guselkumab group and the placebo group. There were significant differences in PASI $75(\mathrm{OR}=3.08,95 \%$ $\mathrm{CI}=2.35$ to 4.06$)$, IGA $0 / 1(\mathrm{OR}=2.79,95 \% \mathrm{CI}=2.17$ to 3.59), and DLQI $0 / 1(\mathrm{OR}=1.86,95 \% \mathrm{CI}=1.50$ to 2.31$)$ between the guselkumab group and the adalimumab group.

3.4. Secondary Outcomes. As shown in Figure 4, there were significant differences in PASI $90(\mathrm{OR}=55.3,95 \% \mathrm{CI}=$ 24.74 to 123.61$)$ and PASI $100(\mathrm{OR}=36.37,95 \% \mathrm{CI}=12.46$ to 106.21) between the guselkumab group and the placebo group. Meanwhile, there were significant differences in PASI $90(\mathrm{OR}=2.66,95 \% \mathrm{CI}=2.14$ to 3.31$)$ and PASI 100 $(\mathrm{OR}=2.28,95 \% \mathrm{CI}=1.63$ to 3.17$)$ between the guselkumab group and the adalimumab group.

3.5. Safety of Guselkumab. As shown in Figure 5, guselkumab was well tolerated and the incidence of AEs (OR $=1.05,95 \%$ $\mathrm{CI}=0.86$ to 1.29$)$ and serious $\mathrm{AEs}(\mathrm{OR}=1.03,95 \% \mathrm{CI}=0.47$ to 2.27) were similar to that of the placebo. There was no statistically significant association of infections (OR $=1.11,95 \%$
$\mathrm{CI}=0.87$ to 1.43$)$ and serious infections $(\mathrm{OR}=0.70,95 \%$ $\mathrm{CI}=0.09$ to 5.42 ) compared with the placebo group. Compared to the adalimumab group, the incidences of AEs $(\mathrm{OR}=0.97,95 \% \mathrm{CI}=0.79$ to 1.19$)$, serious $\mathrm{AEs}(\mathrm{OR}=0.91$, $95 \% \mathrm{CI}=0.44$ to 1.87 ), and infections $(\mathrm{OR}=1.00,95 \%$ $\mathrm{CI}=0.78$ to 1.27$)$ and serious infections ( $\mathrm{OR}=0.35,95 \%$ $\mathrm{CI}=0.07$ to 1.74 ) in guselkumab group were not significantly different.

\section{Discussion}

Recently, although TNF inhibitors were been widely used and the traditional treatment strategies of psoriasis were changed, but some refractory patients still might have inhibitor resistance. Meanwhile, studies reported that the intervention of IL-23 in susceptible mice could lead to psoriasis-like lesions, and the expression of IL-23 was elevated in the human psoriasis tissue [17-19], which further testified that IL-23 might be a pathogenic factor of human psoriasis. Blauvelt et al. [19] demonstrated that guselkumab, IL-23p19 inhibitor, was effective in treating plaque psoriasis. In this meta-analysis, the PASI, IGA, and DLQI were used as the main efficacy indicators and AEs and SAEs as the main safety indicators to comprehensively analyze and compare the efficacy and safety of guselkumab.

In this meta-analysis, there was moderate heterogeneity between the enrolled studies $\left(0 \%<I^{2}<57 \%\right)$; hence, the random effects model was performed. The reason for heterogeneity might be the sample size. The reports by Howard et al. and Nemoto et al. only included several patients, and they did not adjust for the number of participants, which might have increased the probability of smaller $P$ values and narrower CIs between the guselkumab and placebo groups. The results in this study showed that there were significant positive benefits for the guselkumab on the PASI 75, 


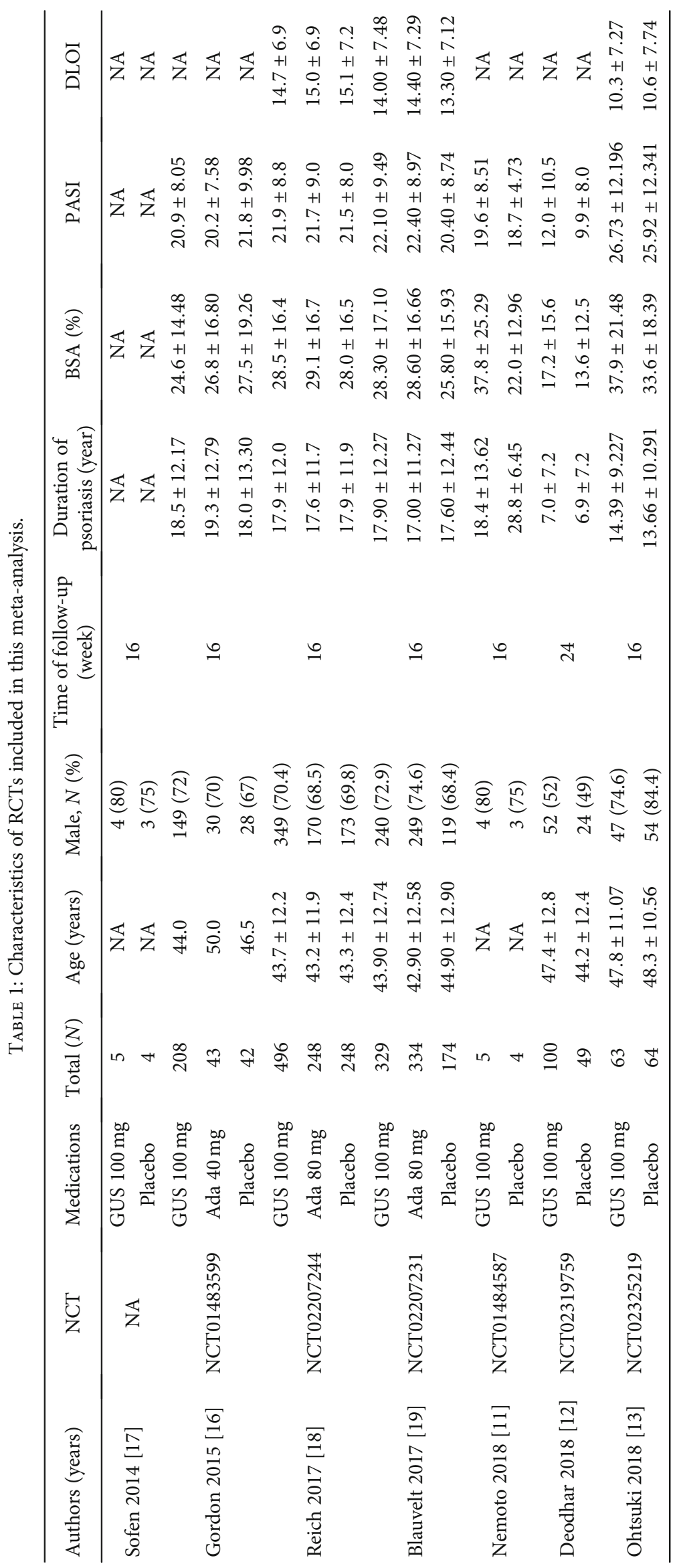




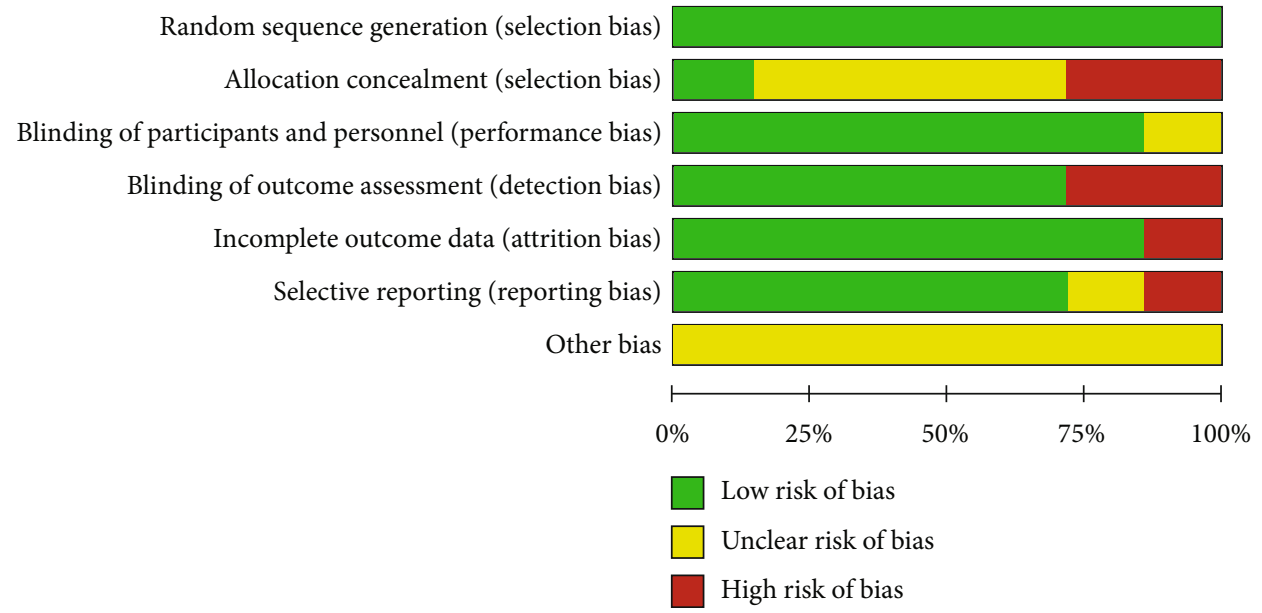

FIGURE 2: Risk of bias summary.

\begin{tabular}{|c|c|c|c|c|c|c|c|c|}
\hline \multirow{2}{*}{$\frac{\text { Study or subgroup }}{\text { Blauvelt } 2017}$} & \multicolumn{2}{|c|}{ Guselkumab } & \multicolumn{2}{|c|}{ Placebo } & Weight & $\begin{array}{c}\text { Odds ratio } \\
\text { M-H, random, } 95 \% \mathrm{CI} \\
\end{array}$ & \multicolumn{2}{|c|}{$\begin{array}{c}\text { Odds ratio } \\
\text { M-H, random, } 95 \% \mathrm{CI} \\
\end{array}$} \\
\hline & 300 & 329 & 10 & 174 & $22.3 \%$ & $169.66[80.66,356.84]$ & & -7 \\
\hline Deodhar 2018 & 77 & 98 & 6 & 48 & $18.6 \%$ & $25.67[9.61,68.53]$ & & $\rightarrow$ \\
\hline Gordon 2015 & 33 & 42 & 2 & 42 & $11.3 \%$ & $73.33[14.81,363.22]$ & & \\
\hline Nemoto 2018 & 3 & 5 & 0 & 4 & $3.6 \%$ & $12.60[0.45,356.37]$ & & \\
\hline Ohtsuki 2018 & 53 & 63 & 4 & 64 & $15.4 \%$ & $79.50[23.54,268.47]$ & & $\pi$ \\
\hline Reich 2017 & 428 & 496 & 20 & 248 & $25.7 \%$ & $71.75[42.50,121.15]$ & & \\
\hline Sofen 2014 & 1 & 4 & 0 & 2 & $3.2 \%$ & $2.14[0.06,77.54]$ & & \\
\hline Total $(95 \% \mathrm{CI})$ & & 1037 & & 582 & $100.0 \%$ & $61.37[31.15,120.91]$ & & $<$ \\
\hline \multicolumn{9}{|l|}{ Total events } \\
\hline $\begin{array}{l}\text { Heterogeneity: tau } \\
\text { Test for overall effec }\end{array}$ & $\begin{array}{l}; \text {;chi }^{2} \\
=1190\end{array}$ & $\begin{array}{l}4.11, \mathrm{df}= \\
<0.0000\end{array}$ & $=6(P=0$ & $.03) ; 1$ & & 0.0 & 0.1 & 10 \\
\hline
\end{tabular}

\begin{tabular}{|c|c|c|c|c|c|c|c|}
\hline \multirow{2}{*}{$\begin{array}{c}\text { Study or subgroup } \\
\text { Blauvelt } 2017\end{array}$} & \multicolumn{2}{|c|}{$\begin{array}{l}\text { Guselkumab } \\
\text { Events }\end{array}$} & \multicolumn{2}{|c|}{$\begin{array}{l}\text { Adalimumab } \\
\text { Events Total }\end{array}$} & \multirow{2}{*}{$\begin{array}{l}\text { Weight } \\
36.3 \%\end{array}$} & \multirow{2}{*}{$\begin{array}{l}\text { Odds ratio } \\
\text { M-H, fixed, } 95 \% \text { CI }\end{array}$} & $\begin{array}{c}\text { Odds ratio } \\
\text { M-H, fixed, } 95 \% \mathrm{CI}\end{array}$ \\
\hline & 300 & 329 & 244 & 334 & & & $\pi$ \\
\hline Gordon 2015 & 33 & 42 & 30 & 43 & $10.8 \%$ & $1.59[0.59,4.25]$ & \\
\hline Reich 2017 & 428 & 496 & 170 & 248 & $52.9 \%$ & $2.89[1.99,4.18]$ & - \\
\hline Total $(95 \% \mathrm{CI})$ & & 867 & & 615 & $100.0 \%$ & $3.08[2.35,4.06]$ & $\bullet$ \\
\hline Total events & 761 & & 444 & & & & \\
\hline $\begin{array}{l}\text { Heterogeneity: chi } \\
\text { Test for overall effec }\end{array}$ & $\begin{array}{l}\mathrm{df}=2(P) \\
.06(P<\end{array}$ & $\begin{array}{l}0.26) ; I^{2} \\
00001)\end{array}$ & & & & & 1 \\
\hline
\end{tabular}

\begin{tabular}{lccccccc|c}
\hline \multicolumn{10}{c}{} \\
IGA 0/1
\end{tabular}

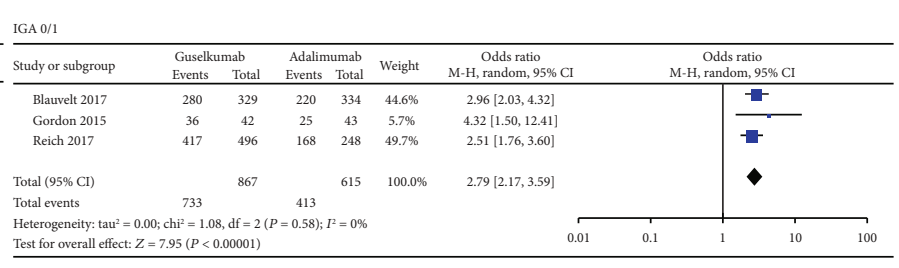
Test for overall effect: $Z=22.33(P<0.00001)$
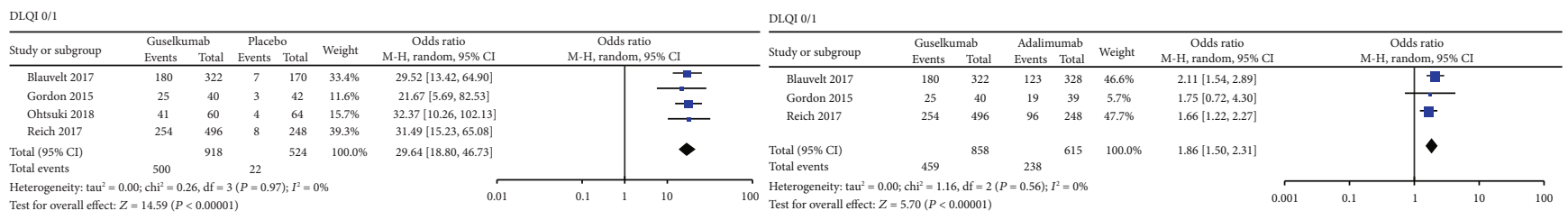

FIgURE 3: Primary efficacy outcomes of guselkumab in the treatment of plaque psoriasis versus placebo or adalimumab. PASI: Psoriasis Area and Severity Index; IGA: Investigator's Global Assessment; DLQI: Dermatology Life Quality Index.

PASI 90, PASI 100, IGA 0/1, and DLQI 0/1 compared with placebo or adalimumab. It was consistent with the conclusions of several reviews [20-22]. The incidences of AEs, serious AEs, and infections and serious infections were not significantly different between the groups. The result of infections was inconsistent with some studies. The study of $\mathrm{Xu}$ et al. [23] showed that guselkumab might increase the incidence of infections compared with placebo, but there were not existing research reports that the infections had evolved into a serious infection or other SAEs. It was consistent with this meta-analysis. Therefore, the results showed that guselkumab was likely the very efficacious treatment. In previous studies, guselkumab had not been directly investigation, and the results of this study might provide some indirect evidence for clinical application. But the conclusions still needed to be verified by RCTs with a lager sample size. Recent researches by Ohtsuki et al. [24] and Reich et al. [25] showed that guselkumab was effective and safety for the treatment of moderate-tosevere plaque psoriasis. It was to be regretted that the studies were not included, because they had very serious heterogeneous.

Adalimumab is a biological agent targeted at TNF- $\alpha$, which has been proved to have good efficacy in other autoimmune diseases [26]. Although its efficacy in psoriasis was better than placebo, it was unknown compared 
PASI 90

\begin{tabular}{|c|c|c|c|c|c|c|c|}
\hline \multirow{2}{*}{$\frac{\text { Study or subgroup }}{\text { Blauvelt } 2017}$} & \multicolumn{2}{|c|}{ Guselkumab } & \multicolumn{2}{|c|}{$\begin{array}{l}\text { Placebo } \\
\end{array}$} & Weight & $\begin{array}{c}\text { Odds ratio } \\
\text { M-H, random, } 95 \% \mathrm{CI} \\
\end{array}$ & $\begin{array}{c}\text { Odds ratio } \\
\mathrm{M}-\mathrm{H} \text {, random, } 95 \% \mathrm{CI} \\
\end{array}$ \\
\hline & 241 & 329 & 10 & 174 & $25.7 \%$ & $92.57[36.80,232.81]$ & $\Rightarrow$ \\
\hline Deodhar 2018 & 65 & 98 & 5 & 48 & $20.2 \%$ & $29.55[8.54,102.25]$ & $=$ \\
\hline Gordon 2015 & 26 & 42 & 1 & 42 & $10.8 \%$ & $66.63[8.33,532.86]$ & \\
\hline Nemoto 2018 & 1 & 5 & 0 & 4 & $4.8 \%$ & $3.00[0.09,97.17]$ & \\
\hline Ohtsuki 2018 & 347 & 496 & 6 & 248 & $27.4 \%$ & $93.93[40.86,215.94]$ & $\Rightarrow$ \\
\hline Reich 2017 & 44 & 63 & 0 & 64 & $6.7 \%$ & $294.38[17.32,5003.39]$ & - \\
\hline Sofen 2014 & 1 & 4 & 0 & 2 & $4.4 \%$ & $2.14[0.06,77.54]$ & \\
\hline Cl) & & 1037 & & 582 & $100.0 \%$ & $55.30[24.74,123.61]$ & \\
\hline
\end{tabular}

Heterogeneity: tau $^{2}=0.43 ;$ chi' $=10.56, \mathrm{df}=6(P=0.10) ; I^{2}=43 \%$
Test for overall effect: $Z=9.78(P<0.00001)$

PASI 100

\begin{tabular}{|c|c|c|c|c|c|c|c|}
\hline \multirow{2}{*}{$\frac{\text { Study or subgroup }}{\text { Blauvelt } 2017}$} & \multicolumn{2}{|c|}{ Guselkumab } & \multicolumn{2}{|c|}{ Placebo } & \multirow{2}{*}{$\begin{array}{r}\text { Weight } \\
18.9 \%\end{array}$} & \multirow{2}{*}{$\begin{array}{c}\begin{array}{c}\text { Odds ratio } \\
\text { M-H, random, } 95 \% \mathrm{CI}\end{array} \\
103.30[14.29,746.85]\end{array}$} & $\begin{array}{c}\text { Odds ratio } \\
\text { M-H, random, } 95 \% \mathrm{CI}\end{array}$ \\
\hline & 123 & 329 & 1 & 174 & & & \\
\hline Deodhar 2018 & 39 & 98 & 3 & 48 & $31.0 \%$ & $9.92[2.88,34.15]$ & 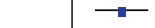 \\
\hline Gordon 2015 & 14 & 42 & 0 & 42 & $11.1 \%$ & $43.25[2.48,754.27]$ & \\
\hline Ohtsuki 2018 & 17 & 63 & 0 & 64 & $11.2 \%$ & $48.55[2.85,827.87]$ & \\
\hline Reich 2017 & 169 & 496 & 2 & 248 & $27.7 \%$ & $63.57[15.62,258.78]$ & \\
\hline Total $(95 \% \mathrm{CI})$ & & 1028 & & 576 & $100.0 \%$ & $36.37[12.46,106.21]$ & \\
\hline Total events & 362 & & 6 & & & & \\
\hline
\end{tabular}

PASI 90

\begin{tabular}{|c|c|c|c|c|c|c|c|}
\hline \multirow{2}{*}{$\frac{\text { Study or subgroup }}{\text { Blauvelt } 2017}$} & \multicolumn{2}{|c|}{ Guselkumab } & \multicolumn{2}{|c|}{ Adalimumab } & Weight & $\begin{array}{c}\text { Odds ratio } \\
\mathrm{M}-\mathrm{H} \text {, random, } 95 \% \mathrm{CI} \\
\end{array}$ & $\begin{array}{c}\text { Odds ratio } \\
\text { M-H, random, } 95 \% \mathrm{C} \\
\end{array}$ \\
\hline & 241 & 329 & 166 & 334 & $45.3 \%$ & $2.77[2.00,3.84]$ & - \\
\hline Gordon 2015 & 26 & 42 & 19 & 43 & $6.4 \%$ & $2.05[0.86,4.88]$ & \\
\hline Reich 2017 & 347 & 496 & 116 & 248 & $48.3 \%$ & $2.65[1.93,3.63]$ & 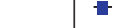 \\
\hline Total $(95 \% \mathrm{CI})$ & & 867 & & 625 & $100.0 \%$ & $2.66[2.14,3.31]$ & $\checkmark$ \\
\hline & 614 & & 301 & & & & \\
\hline
\end{tabular}

Test for overall effect: $Z=8.77(P<0.00001)$

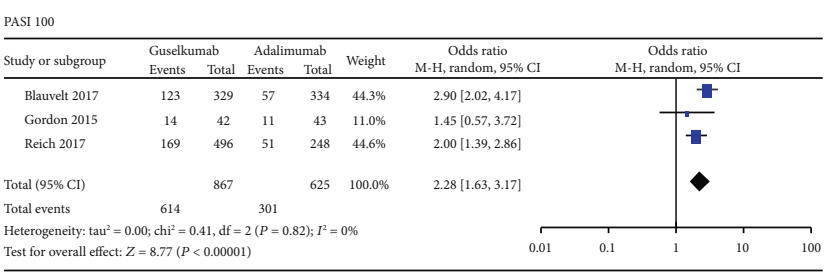

FIGURE 4: Secondary efficacy outcomes of guselkumab in the treatment of plaque psoriasis versus placebo or adalimumab. PASI: Psoriasis Area and Severity Index.
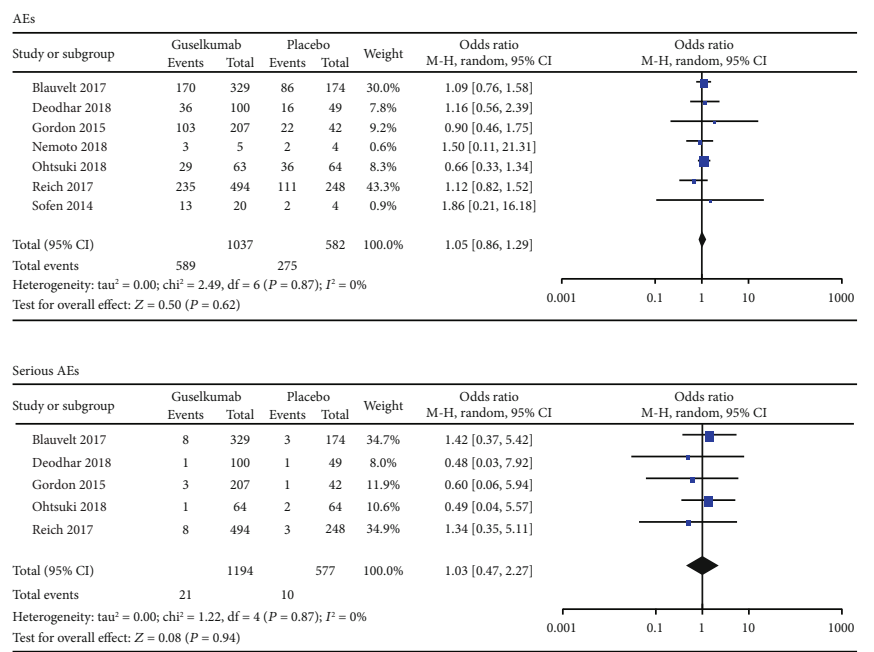

作

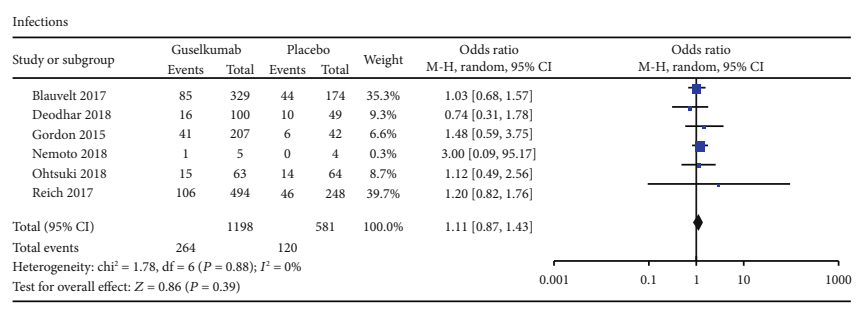

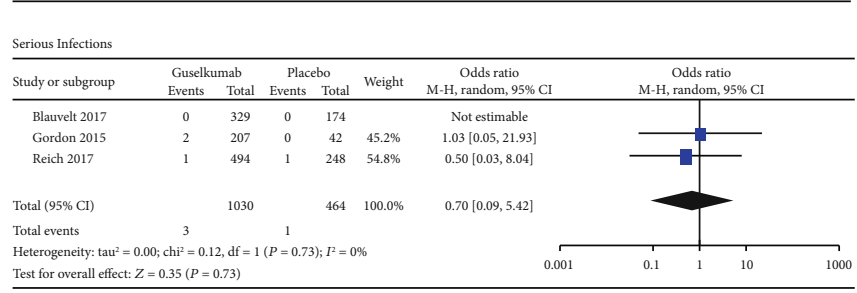
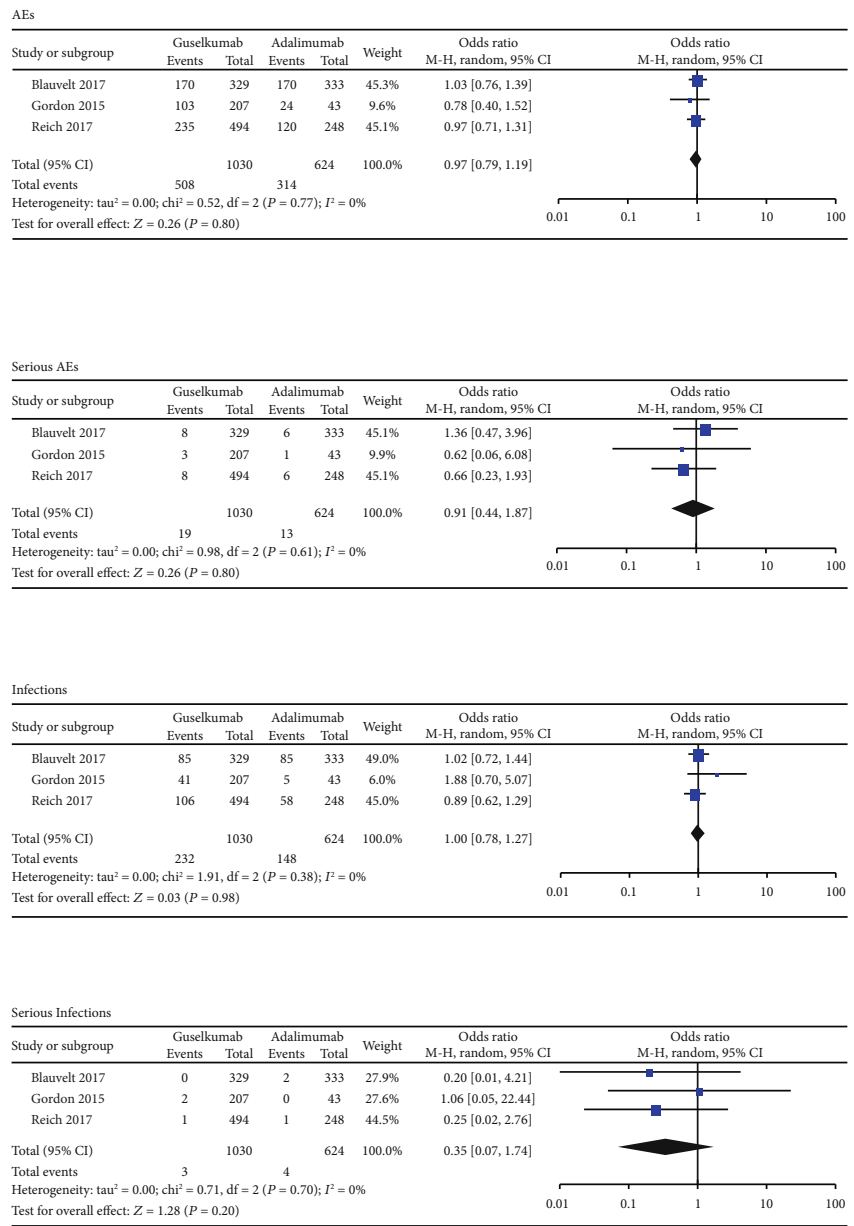

FIGURE 5: Safety outcomes of guselkumab in the treatment of plaque psoriasis versus placebo or adalimumab. AEs: adverse events; SAEs: serious adverse events.

with guselkumab. The results of this meta-analysis showed guselkumab had superior efficacy to adalimumab in the achievements of PASI 75, PASI 90, PASI 100, IGA 0/1, and DLQI 0/1, but there were no significant differences in incidence rates of safety indicators. They might have similar tolerances. 
Sensitivity analyses that excluded low-quality trials and studies that exclusively enrolled patients with particular medical conditions did not alter these results. It was indicated that our results were statistically robust. Publication bias was not reported because the number of trials was less than 10 for each comparison. There were several limitations in this study. First, some comparisons and analyses could not be done, because the RCTs about them have not been done or published. Second, long-term safety needed to be further confirmed by long-term clinical trials. Finally, the quantity and sample size of the literatures were not perfect; the data were deficiency. Accordingly, the efficacy and safety of guselkumab needed to be discussed later.

In this meta-analysis, data were updated compared with prior reports. The subgroup analysis was not performed in this meta-analysis because the included trials were limited. This meta-analysis showed that guselkumab had good efficacy and safety in patients with moderate-to-severe plaque psoriasis and had a better efficacy than adalimumab without other adverse events. But long-term safety and the maintenance of efficacy remained to be determined; future studies should focus more on long-term follow-up.

\section{Data Availability}

This article does not involve any basic experiments and clinical investigations. It only requires a database search, and these data are public and free.

\section{Conflicts of Interest}

All authors declare no conflicts of interest.

\section{References}

[1] R. Talaee, Z. Hajheydari, A. Y. Moghaddam, S. A. Moraveji, and B. F. Ravandi, "Prevalence of oral mucosal lesions and their association with severity of psoriasis among psoriatic patients referred to dermatology clinic: a cross-sectional study in Kashan/Iran," Open Access Macedonian Journal of Medical Sciences, vol. 5, no. 7, pp. 978-982, 2017.

[2] D. Kumar, J. P. Rajguru, D. Maya, P. Suri, S. Bhardwaj, and N. D. Patel, "Update on psoriasis: a review," Journal of Family Medicine and Primary Care, vol. 9, no. 1, pp. 20-24, 2020.

[3] K. L. Goff, C. Karimkhani, L. N. Boyers et al., "The global burden of psoriatic skin disease," The British Journal of Dermatology, vol. 172, no. 6, pp. 1665-1668, 2015.

[4] G. E. Fragoulis, S. Siebert, and I. B. McInnes, "Therapeutic targeting of IL-17 and IL-23 cytokines in immune-mediated diseases," Annual Review of Medicine, vol. 67, no. 1, pp. 337-353, 2016.

[5] V. Oji and T. A. Luger, "The skin in psoriasis: assessment and challenges," Clinical and Experimental Rheumatology, vol. 33, no. 5, Supplement 93, pp. S14-S19, 2015.

[6] A. Nadeem, S. F. Ahmad, N. O. Al-Harbi et al., "Bruton's tyrosine kinase inhibitor suppresses imiquimod-induced psoriasislike inflammation in mice through regulation of IL-23/IL-17A in innate immune cells," International Immunopharmacology, vol. 80, p. 106215, 2020.
[7] M. Marovt, P. B. Marko, M. Pirnat, and R. Ekart, "Effect of biologics targeting interleukin-23/-17 axis on subclinical atherosclerosis: results of a pilot study," Clinical and Experimental Dermatology, vol. 45, no. 5, pp. 560-564, 2020.

[8] B. Filiz, M. Yildirim, K. H. Öztürk et al., "Evaluation of interleukin-23 receptor (IL-23R) gene polymorphisms and serum IL-23 levels in patients with psoriasis," Turkish Journal Of Medical Sciences, vol. 49, no. 5, pp. 1386-1394, 2019.

[9] J. J. Crowley, R. B. Warren, and J. C. Cather, "Safety of selective IL-23p19 inhibitors for the treatment of psoriasis," Journal of the European Academy of Dermatology and Venereology, vol. 33, no. 9, pp. 1676-1684, 2019.

[10] J. J. Wu and W. C. Valdecantos, "Adalimumab in chronic plaque psoriasis: a clinical guide," Journal of Drugs in Dermatology, vol. 16, no. 8, pp. 779-790, 2017.

[11] O. Nemoto, K. Hirose, S. Shibata, K. Li, and H. Kubo, "Safety and efficacy of guselkumab in Japanese patients with moderate-to-severe plaque psoriasis: a randomized, placebocontrolled, ascending-dose study," The British Journal of Dermatology, vol. 178, no. 3, pp. 689-696, 2018.

[12] A. Deodhar, A. B. Gottlieb, W. H. Boehncke et al., "Efficacy and safety of guselkumab in patients with active psoriatic arthritis: a randomised, double-blind, placebo-controlled, phase 2 study," The Lancet, vol. 391, no. 10136, pp. 22132224, 2018.

[13] M. Ohtsuki, H. Kubo, H. Morishima, R. Goto, R. Zheng, and H. Nakagawa, "Guselkumab, an anti-interleukin-23 monoclonal antibody, for the treatment of moderate to severe plaquetype psoriasis in Japanese patients: efficacy and safety results from a phase 3, randomized, double-blind, placebocontrolled study," The Journal of Dermatology, vol. 45, no. 9, pp. 1053-1062, 2018.

[14] K. A. Papp, M. Yang, M. Sundaram et al., "Comparison of adalimumab and etanercept for the treatment of moderate to severe psoriasis: an indirect comparison using individual patient data from randomized trials," Value in Health, vol. 21, no. 1, pp. 1-8, 2018.

[15] L. M. Sawyer, K. Malottki, C. Sabry-Grant et al., "Assessing the relative efficacy of interleukin-17 and interleukin-23 targeted treatments for moderate-to-severe plaque psoriasis: a systematic review and network meta-analysis of PASI response," PLoS One, vol. 14, no. 8, article e0220868, 2019.

[16] K. B. Gordon, K. C. Duffin, R. Bissonnette et al., "A phase 2 trial of guselkumab versus adalimumab for plaque psoriasis," The New England Journal of Medicine, vol. 373, no. 2, pp. 136-144, 2015.

[17] H. Sofen, S. Smith, R. T. Matheson et al., "Guselkumab (an IL23-specific $\mathrm{mAb}$ ) demonstrates clinical and molecular response in patients with moderate-to-severe psoriasis," The Journal of Allergy and Clinical Immunology, vol. 133, no. 4, pp. 1032-1040, 2014.

[18] K. Reich, A. W. Armstrong, P. Foley et al., "Efficacy and safety of guselkumab, an anti-interleukin-23 monoclonal antibody, compared with adalimumab for the treatment of patients with moderate to severe psoriasis with randomized withdrawal and retreatment: results from the phase III, double-blind, placeboand active comparator-controlled VOYAGE 2 trial," Journal of the American Academy of Dermatology, vol. 76, no. 3, pp. 418431, 2017.

[19] A. Blauvelt, K. A. Papp, C. E. Griffiths et al., "Efficacy and safety of guselkumab, an anti-interleukin-23 monoclonal antibody, compared with adalimumab for the continuous 
treatment of patients with moderate to severe psoriasis: results from the phase III, double-blinded, placebo- and active comparator-controlled VOYAGE 1 trial," Journal of the American Academy of Dermatology, vol. 76, no. 3, pp. 405-417, 2017.

[20] J. R. Chan, W. Blumenschein, E. Murphy et al., "IL-23 stimulates epidermal hyperplasia via TNF and IL-20R2-dependent mechanisms with implications for psoriasis pathogenesis," The Journal of Experimental Medicine, vol. 203, no. 12, pp. 2577-2587, 2006.

[21] M. Elliott, J. Benson, M. Blank et al., "Ustekinumab: lessons learned from targeting interleukin-12/23p40 in immunemediated diseases," Annals of the New York Academy of Sciences, vol. 1182, no. 1, pp. 97-110, 2009.

[22] A. A. Levin and A. B. Gottlieb, "Specific targeting of interleukin-23p19 as effective treatment for psoriasis," Journal of the American Academy of Dermatology, vol. 70, no. 3, pp. 555-561, 2014.

[23] S. Xu, X. Zhang, M. Pan, Z. Shuai, S. Xu, and F. Pan, “Treatment of plaque psoriasis with IL-23p19 blockers: a systematic review and meta-analysis," International Immunopharmacology, vol. 75, p. 105841, 2019.

[24] M. Ohtsuki, H. Fujita, M. Watanabe et al., "Efficacy and safety of risankizumab in Japanese patients with moderate to severe plaque psoriasis: results from the SustaIMMphase 2/3 trial," The Journal of Dermatology, vol. 46, no. 8, pp. 686-694, 2019.

[25] K. Reich, M. Gooderham, D. Thaçi et al., "Risankizumab compared with adalimumab in patients with moderate-to-severe plaque psoriasis (IMMvent): a randomised, double-blind, active-comparator-controlled phase 3 trial," The Lancet, vol. 394, no. 10198, pp. 576-586, 2019.

[26] K. Papp, D. Thaçi, D. Marcoux et al., "Efficacy and safety of adalimumab every other week versus methotrexate once weekly in children and adolescents with severe chronic plaque psoriasis: a randomised, double-blind, phase 3 trial," The Lancet, vol. 390, no. 10089, pp. 40-49, 2017. 